\title{
Applications of Visible Light Path Laser Projector
}

\author{
Nobuchika Sakata, Shu Okamoto, and Shogo Nishida
}

Division of Systems Science and Applied Informatics Graduate School of Engineering Science, Osaka University, Machikaneyama-cho 1-3, Toyonaka city, Osaka 560-8531 Japan \{sakata, okamoto, nishida\}@nishilab.sys.es.osaka-u.ac.jp

\begin{abstract}
We proposed and developed a method to visualize the light path of a laser with jetting mists along light axis of a laser pointer. The estimation accuracy for the position of a laser spot occluded behind an object was improved when the light path of the laser was visualized. In this paper, we propose visible light path laser projector (VLLP) for remote collaboration. VLLP is equipped with a laser projector and a mist generator. With the laser projector, VLLP can instruct with not only a laser spot but also simple line drawings. Furthermore, we propose several VLLP application prototypes.
\end{abstract}

Keywords: Remote collaboration, Visible Light Path Laser, VLLP, Laser projector.

\section{Introduction}

Work conducted by a local worker under the instructions of a remote instructor is called remote collaboration. Using a telecommunication terminal, the remote instructor and the local worker transmit and receive sounds and videos to accomplish their work since they can not share voices and views directly. On the other hand, a worker and an instructor sometimes communicate regarding objects and places in real work spaces in local collaborative works [1-3]. To conduct such communication smoothly, a support system sends the remote instructor's instructions including the place of the work to the local worker. In some research, a teleoperated laser pointer or laser projector is adopted as a means of sending such instruction [4-8] This method enables a remote collaboration system to be realized with a compact device. The teleoperated laser pointer has been adopted as a tool to point at a target of a work for compact systems [6,7]. However, although the instructor can watch the laser spot, this method can be difficult for workers observing the laser spot when it is occluded behind the object being pointed at or other obstacles. Research has shown that laserpointing enables smooth remote collaboration for simple tasks, such as selecting and specifying real-world targets. In addition, a laser spot not only provides awareness information about a remote instructor's point of view, but also links a real-world position and virtual information to a local worker. Therefore, the remote collaboration cannot be conducted smoothly when the laser spot providing important information is hidden from the local worker's sight. The proposed method thus allows for smooth remote collaboration by realizing $3 \mathrm{D}$ directional expression to enable a worker to 
estimate the position of a laser spot from the visualized light path, even if the laser spot is occluded.

Originally, this research aims to support remote collaboration, including instructions identifying real objects with a compact device. In previous research, we proposed a new method for pointing to objects for remote collaboration support devices and provides an assessment of the method. The method used 3D directional expression, which is similar to using a pointing stick, by visualizing the light path of a laser pointer. In user test we conducted, we found that with this method, a worker can be expected to estimate the position of an occluded laser spot from the visualized light path. In this paper, applying knowledge of visible light path laser pointer, we propose visible light path laser projector (VLLP) for remote collaboration. Furthermore, using 3D directional expression of VLLP, we propose not only applications of remote collaboration, but also several VLLP application prototypes.

\section{Related Works}

The teleoperated laser pointer is adopted in some research as a pointing tool for remote collaboration. Telepointer [4] is a wearable device mounted on the chest. Telepointer is equipped with a fixed camera and a teleoperated laser pointer which enables remote collaboration targeting objects in real-world. A Wearable Active Camera with a Laser pointer (WACL) [7] is a hands, head, and eye-free wearable device mounted on a worker's shoulder. WACL is comprised of a camera and a laser pointer on a biaxial camera platform. Sakata et al. conducted an experiment to compare settings when a laser spot is projected and not projected on targets [9]. The results showed that the laser spot played an important role, named the visual link, linking information shown on a chest worn display and the real-world position the information regarded. GestureCam [5] is not a wearable device but a device placed in a work space. GestureCam has a camera mounting a laser pointer on it. An instructor can pan and tilt the laser pointer on the camera to point at real-world objects. CTerm [6] is also a device placed in a work space. CTerm is equipped with a camera and a laser pointer which are remotely controlled.

Some studies have realized systems to convey gestural expression to remote sites. These systems can express 3D direction. GestureMan [10] is a system equipped with not only a teleoperated laser pointer but also a robot head and a robot arm. The robot head and the robot arm trace the motion of the remote instructor. Therefore, GestureMan is assumed to be able to convey the target of instruction even if the laser spot is occluded. However, the robot arm and the robot head prevent the system from being reduced in size for compactness because the robot arm and the robot head require a certain size to express gestures and orientation. Telecommunicator T2 [11] is a wearable communication system with gesture expressions. Telecommunicator T2 has a teleoperated camera and a compact arm. Using this system, a remote person can communicate a wearer with gestures of the arm. However, the system does not aim to support remote collaboration with instructions regarding real-world objects. Moreover, the size of the arm is limited to enable mounting on the shoulder. Therefore, pointing with Telecommunicator T2 is not as accurate as using a laser pointer. Real World Video Avatar [12] is a telecommunication system which can 
convey human gestures. The system shows images at all angles by spinning a large display, on which a filter enhancing the directivity is attached, with the images and the angle of the display synchronized. Although Real World Video Avatar has rich expression, the device is large in scale.

As described above, remote collaboration systems with rich gestural expressions are large-scale, although they are assumed to be able to convey instructions regarding real-world objects without relying upon a laser spot. Thus, there is a trade-off between rich 3D directional expression and compactness. There has been no research aimed at realizing rich $3 \mathrm{D}$ directional expression with a compact device.

\section{Visible Light Path Laser Projector (VLLP)}

Named the Visible Light path Laser Projector (VLLP), for remote collaboration is being constructed. VLLP is equipped with a laser projector and a mist generator as shown in Fig. 1. With the laser projector, VLLP can instruct with not only a laser spot but also simple line drawings. We describe visible light path as main function of VLLP in section 3.1.

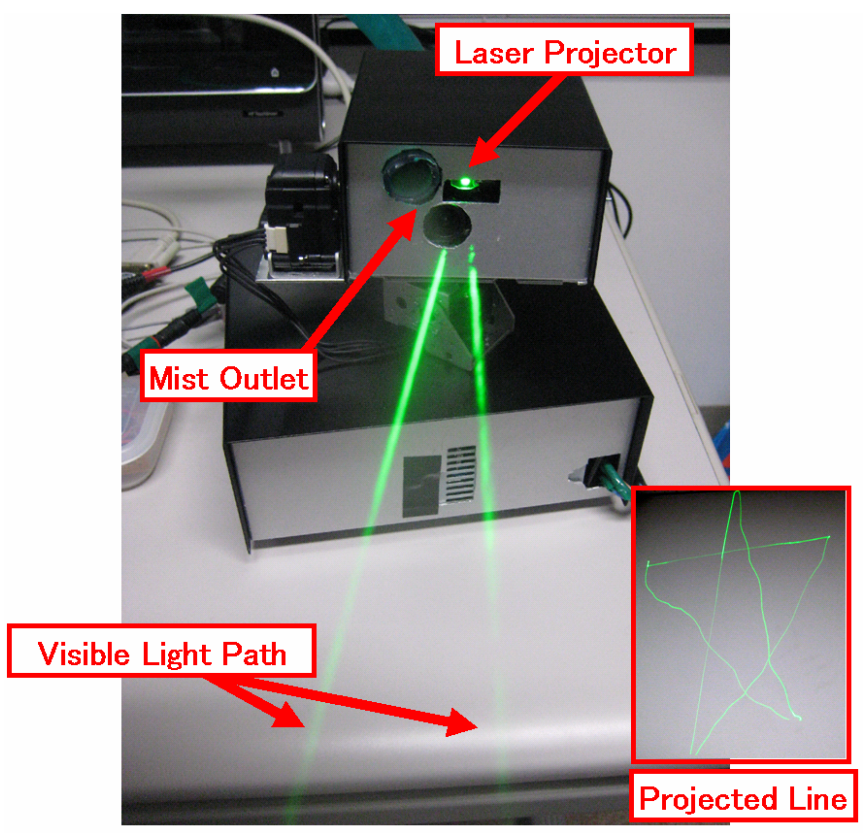

Fig. 1. Visible light path laser projector

\subsection{Visible Light Path Laser Pointer}

The light path of a laser is visualized as a bright line when the laser is scattered by particles. Scattering a high-power laser with dust is a possible method for visualizing the light path of a laser. However, a high-power laser is not suitable for remote 
collaboration since it can harm a worker. Although scattering a low-power laser by filling up the workspace with mist is harmless, time is required to fill up the workspace with an artificial mist even if a large-size device is applied. Therefore, we suggests a harmless method which requires neither a large-scale device, nor a long wait time. The proposed method scatters a laser with an artificially generated mist jetted in the direction of the laser with a fan. This allows a reduction in the necessary amount of mist and reduces the required size of the device compared with filling up the workspace with mist. Moreover, this method has low danger since the method can visualize the light path of the laser even at low power. An analogous method was suggested by Shimizu and Takaguchi, who proposed shooting a "light arrow" by shooting smoke from an air cannon along a laser [13]. Their method has merit in that less smoke is required and the smoke can reach farther than the method proposed here. However, the light path is difficult to watch since it is intermittent. In addition, an air cannon is not suitable for mounting on a compact device. For these reasons the method proposed here uses not an air cannon but a fan.

A visible light path laser pointer was developed experimentally by applying the suggested method and is shown in Fig. 2. The device consists of a mist generator and a laser pointer. A mist generated by the mist generator is transferred to a hose by a fan, and then jets from the hose end. A laser pointer is mounted on the hose end along the direction of jetted mists, so the laser goes through the mist. The mist generator is equipped with two ultrasound atomizer HM-2412 (Honda Electronics Co., Ltd.). One HM-2412 can atomize approximately $250 \mathrm{ml} / \mathrm{h}$ of water. The size of a mist particle is approximately $3 \mu \mathrm{m}$. The size of one circuit board is $45 \times 70 \mathrm{~mm} 2$, and the size of one oscillator for the mist generator is $42 \times 30 \times 13 \mathrm{~mm} 3$. However, there are many

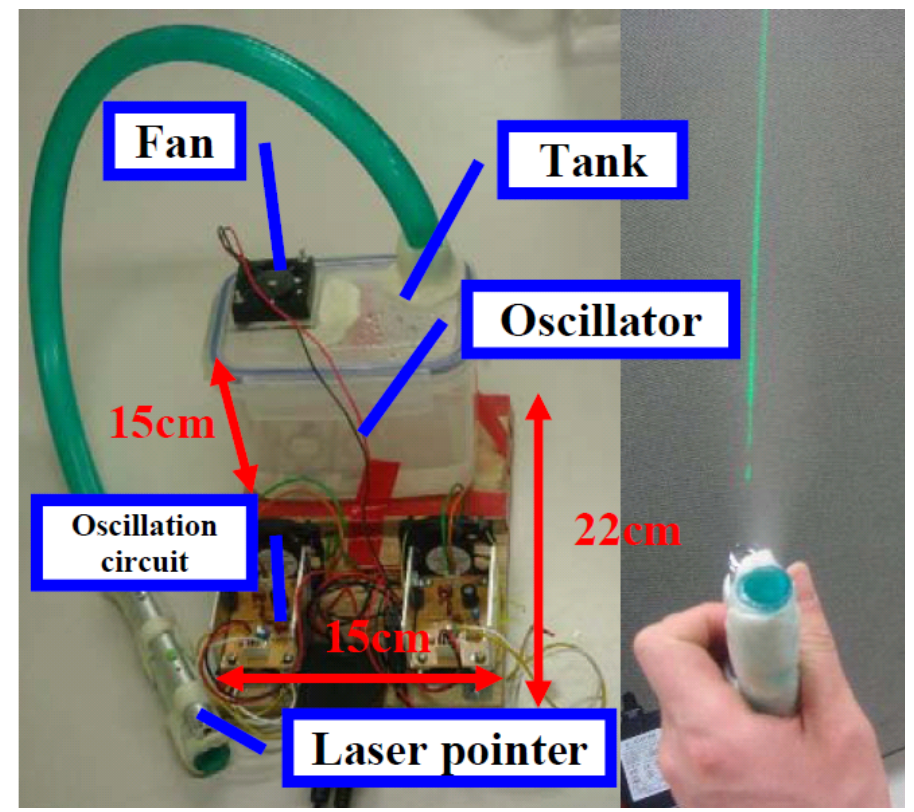

Fig. 2. Laser pointer with jetting mist along laser light path 
products smaller than HM-2412 but provide the same function. The laser pointer is a commercially available product with an on/off push switch. The laser is green with a wavelength of $532 \mathrm{~nm}$, maximum output of less than $1 \mathrm{~mW}$, and a beam width of 5 $\mathrm{mm}$. The length from the switch to the end of the laser pointer is $46 \mathrm{~mm}$, and the length from the switch to the end of the hose is $55 \mathrm{~mm}$.

The light path of the visible light path laser pointer with atomized water could be observed under room lights of 460 lx illuminance. The length of the visible part of the path was approximately $1 \mathrm{~m}$. The length was slightly short compared to a pointing stick, probably caused by the ready vaporization of the water mist. To prevent vaporization, fog liquid (FLG1, Stage Evolution), which is a liquid for smoke machines, was atomized. When fog liquid diluted to $20 \%$ with water was atomized, the length of the visible part was extended to approximately $3 \mathrm{~m}$ since the fog liquid vaporized less easily than water. However, the amount of mist decreased because FLG1 was not atomized by HM-2412. As a result, the brightness of the light path decreased compared with the case of water.

Though the light path was bright enough to be easily observed from the front of the laser pointer, the light path was less easy to see from the side. In addition, when the direction of the laser pointer was changed, the visible light path took a short time until the mist followed the movement. The delay was approximately $0.5 .1 \mathrm{~s}$ when water was used. Moreover, in windy circumstances such as outdoors, the mist was blown away, shortening the visible light path.

When a mist is jetted onto a hand, glass, or plastic at a distance of $5 \mathrm{~cm}$ for several minutes, these objects became wet. On the other hand, jetting at a distance of $40 \mathrm{~cm}$ caused little wetting. Therefore, the visible light path laser pointer should not cause any trouble even if in a room with machinery or computers unless mists are jetted onto them directly for a long time. Proper installing of the visible light path laser pointer should prevent moisture from collecting.

The function of the visible light path is similar to a pointing stick. However, a pointing stick cannot point at targets with shorter distances than the length of the pointing stick because the stick may make contact with the target. Moreover, a pointing stick has the danger of contact with a worker or object since a worker may have to work near a device mounted with a pointing stick. Therefore, a pointing stick is not suitable as a device supporting remote collaboration. In contrast, the visible light path laser pointer can point at targets with shorter distances than the length of the visible light path. In addition, the visible light path laser pointer contains no danger of contact with a worker or objects. Therefore, the visible light path laser pointer is suitable for mounting on a compact device supporting remote collaboration.

We conducted user test to confirm effectiveness of visible light path laser pointer. We assume the situation that when an instructor facing a worker tries to point to an object between the worker and the instructor with a laser pointer, the laser spot is likely to be out of worker's line of vision, as shown on the left side of Fig. 3. If the worker can estimate the position of the laser spot from the visualized light path even when the worker cannot see the laser spot, as shown on the right of Fig. 3, the remote collaboration targeting real-world objects can be conducted smoothly. After small user test, the proposed method improves the accuracy when estimating the position of an occluded laser spot. In addition, the proposed method was effective in terms of accuracy when distinguishing an object being pointed at from other objects, even when the laser spot is occluded. 


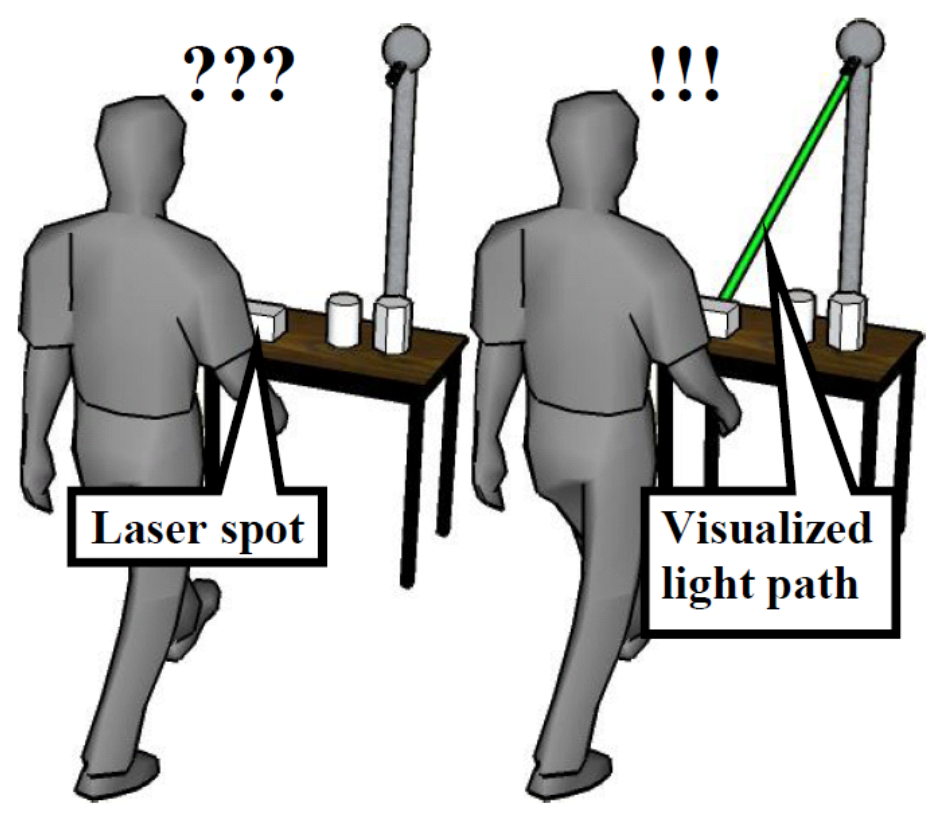

Fig. 3. Laser spot is occluded (left) and light path is visualized (right)

\section{Applications of VLLP}

In this section, we describe two applications of VLLP.

\subsection{Remote Collaboration Terminal for Visual Light Path Laser Projector}

Basically, we suppose to apply VLLP to remote collaboration terminal. We install a microphone, a camera and a speaker to VLLP. We intend to attach a fish-eye camera for observing remote work field widely. With the laser projector, VLLP can instruct with not only a laser spot but also simple line drawings. We assume that combining feature of visible light path and line drawing function with a laser projector, another significant advantage of VLLP is boosted. That is multiple instructions. Teleoperated laser pointer including WACL, telepointer and Gesture Laser can instruct only one point at the same time. On the other hands, teleoperated laser projector can instruct multiple points at the same time. However, laser projector, which is even vector scan type, can not watch the moving laser spot while scanning. For example, remote instructor point three buttons which are spread spatially in work space. And then, remote instructor says "Could you press the buttons?". However, field worker can not find two or three spread button immediately because the instructed button might be existed behind the field worker. In case of applying VLLP, observing visible light path from laser projector, the field worker can sense a direction and place where laser spots exist on. Finally, field worker can detect instructed places easily. We believe this is one big feature of VLLP in remote collaboration. 


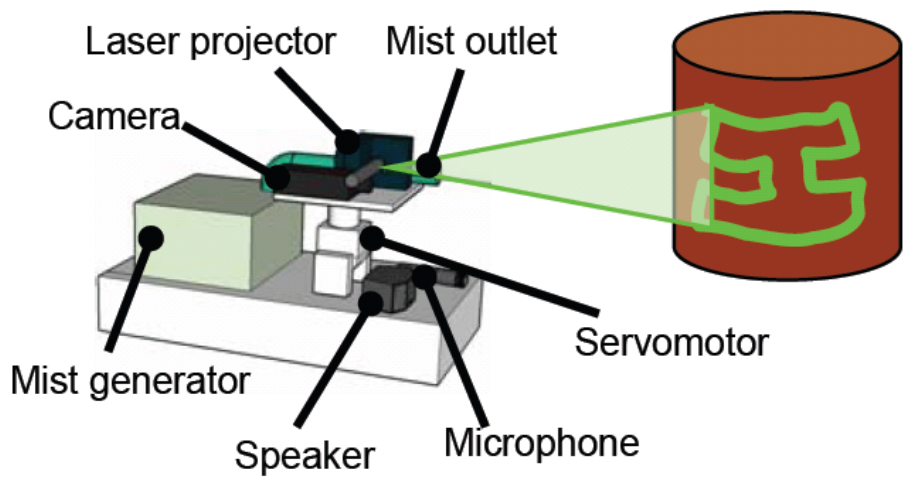

Fig. 4. Visible light path laser projector for remote collaboration

\subsection{Guide Robot Installed VLLLP}

We suppose to use VLLP as one part of guide robot[14][15]. In those researches, various robot body parts such like body size of robot, arms, neck and head take an important role for guide in terms of gestural expressions. Due to this, the size of robot should be kept to generate gestural expressions, consequentially. On the other hands, a risk of clash and spatial storage are increased. For these reasons, we mount VLLP to robot as a part for increasing those gestural expressions (Fig. 5). Using visible light pass as kind of pointing stick, even small robot represents large gestural expressions.

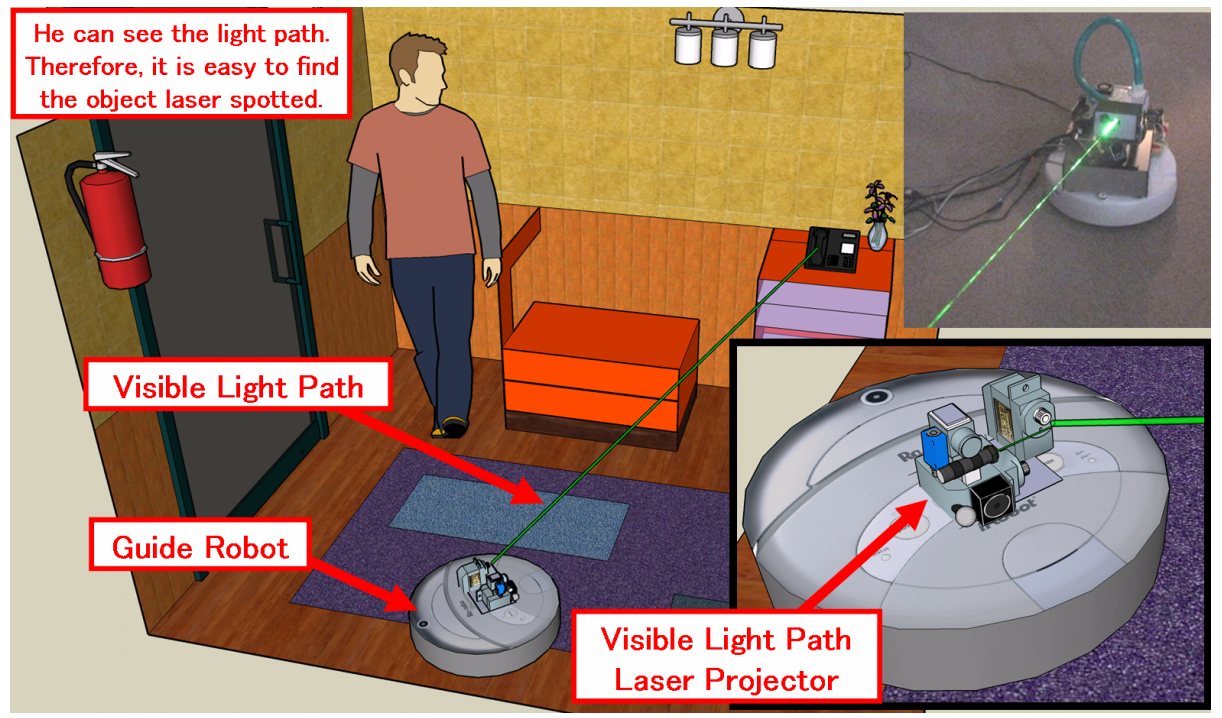

Fig. 5. Visible light path laser projector 


\section{Conclusion}

In this paper, we propose and describe VLLP is equipped with a laser projector and a mist generator. With the laser projector, VLLP can instruct with not only a laser spot but also simple line drawings. Furthermore, we propose two application targets, "Remote collaboration" and "Guide robot". In case of "Remote collaboration", we suggest possibility of multiple instructions. Also, in case of "Guide robot", VLLP can increase gestural expressions instead of neck, head, arms and body. In the future, we develop mobile type VLLP and proposed applications and evaluate features of VLLP.

\section{References}

1. Kuzuoka, H.: Spatial workspace collaboration: a Sharedview video support system for remote collaboration capability. In: CHI 1992, pp. 533-540 (1992)

2. Fussell, S.R., Setlock, L.D., Kraut, R.E.: Effects of head-mounted and scene-oriented video systems on remote collaboration on physical tasks. In: Proc. CHI 2003, pp. 513-520 (2003)

3. Kraut, R.E., Miler, M.D., Siegal, J.: Collaboration in performance of physical tasks: Effects on outcomes and communication. In: CSCW 1996, pp. 57-66 (1996)

4. Mann, S.: Telepointer: Hands-free completely self-contained wearable visual augmented reality without headwear and without any infrastructural reliance. In: ISWC 2000, pp. 177-178 (2000)

5. Kuzuoka, H., Kousuge, T., Tanaka, M.: GestureCam: a video communication system for sympathetic remote collaboration. In: CSCW 1994, pp. 35-43 (1994)

6. Mikawa, M., Matsumoto, M.: Smooth and easy telecommunication using CTerm. In: IEEE SMC 1999, pp. 732-737 (1999)

7. Sakata, N., Kurata, T., Kato, T., Kourogi, M., Kuzuoka, H.: WACL: Supporting telecommunications using wearable active camera with laser pointer. In: ISWC 2003, NY, USA, pp. 53-56 (2003)

8. Palmer, D., Adcock, M., Smith, J., et al.: Annotating with light for remote guidance. In: 2007 conference of CHISIG of Australia on computer-human interaction, pp. 103-110 (2007)

9. Sakata, N., Kurata, T., Kuzuoka, H.: Visual assist with a laser pointer and wearable display for remote collaboration. In: 2nd international conference on Collaboration Technologies (CollabTech. 2006), Tsukuba, Japan, pp. 66-71 (2006)

10. Kuzuoka, H., Furusawa, Y., Kobayashi, N., Yamazaki, K.: Effect on Displaying a Remote Operator's Face on a Media Robot. In: ICCAS 2007, pp. 758-761 (2007)

11. Tsumaki, Y., Fujita, Y., Kasai, A., Sato, C., Nenchev, D.N., Uchiyama, M.: Telecommunicator: A novel robot system for human communications. In: IEEE ROMAN 2002, pp. 35-40 (2002)

12. Tanikawa, T., Suzuki, Y., Hirota, K., Hirose, M.: Real world video avatar: real-time and real-size transmission and presentation of human figure. In: 2005 international conference on Augmented tele-existance, pp. 112-118 (2005)

13. Shimizu, N., Takiguchi, T.: Rokujurossundou. In: VRSJ 8th Annual Conference, p. 289 (2003) (in Japanese)

14. Burgard, A.B., Cremers, D., Fox, D., Hähnel, G., Lakemeyer, D., Schulz, W., Steiner, W., Thrun, S.: Experiences with an interactive museum tour-guide robot. Artificial Intelligence 114(1-2), 3-55 (1999)

15. Kuzuoka, H., Kosaka, J., Yamazaki, K., Yamazaki, A., Suga, Y.: Dual Ecologies of Robot as Communication Media: thoughts on Coordinating Orientations and Projectability. In: CHI 2004, pp. 183-190 (2004) 